محمد مزيان|Mohammed Meziane

\title{
نوعان مـن البشر: تشريح العنصرية العادية
}

The Two Human Species: An Autopsy of Ordinary Racism

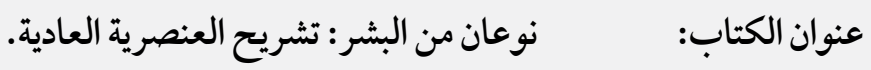

عنوان الكتاب في لغته: Les deux espèces humaines: Autopsie du racisme ordinaire.

$$
\begin{aligned}
& \text { دوني بلوندان Denis Blondin. }
\end{aligned}
$$

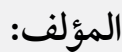

$$
\begin{aligned}
& \text { عاطف المولى. }
\end{aligned}
$$

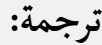

$$
\begin{aligned}
& \text { الدوحة/بيروت: المركز العربي للأبحاث ودراسة السياسات. }
\end{aligned}
$$

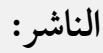

$$
\begin{aligned}
& .2020
\end{aligned}
$$

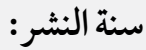

$$
\begin{aligned}
& 296 \text { صفحة. } \\
& \text { عدد الصفحات: }
\end{aligned}
$$


كتابه، فقسّمه إلى ثمانية فصول، فضلاً عن مقدمة

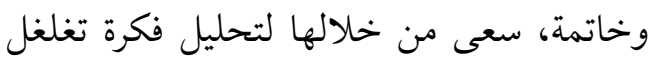

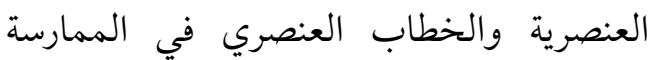

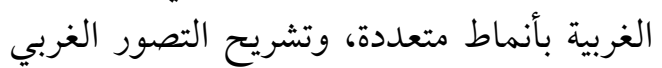

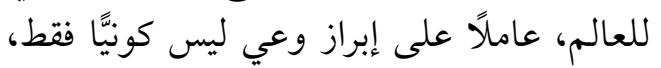

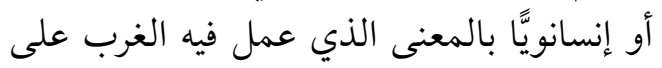

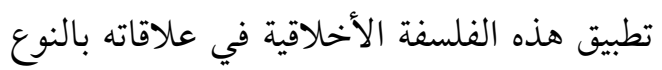
البشري الآخر، بل هو وعيّ مرجعه الإنسان العاقل بomo sapiens

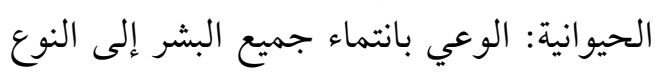
الواحد: الإنسان العاقل (ص 9).

\section{هندسة الكتاب وأفكاره \\ الرئيسة}

يسلّط الفصل الأول "رجل الشيربا المغمور"

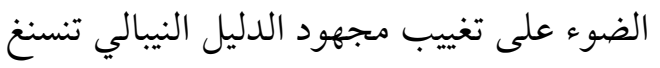

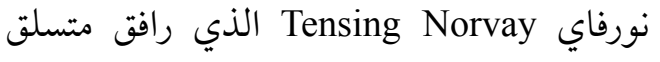
الجبال إدموند هيلاري Edmund Hillary عند بلوغه قمة إفرست عام 1953، فخلّد التاريخ

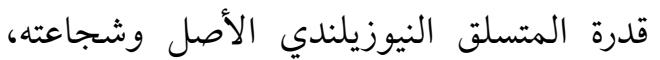
في حين أهمل النيبالي رغم أنه لا يقلّ شجاعة الاصن

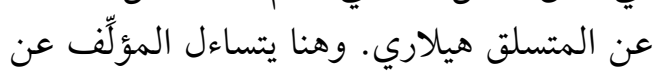

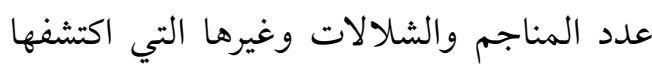

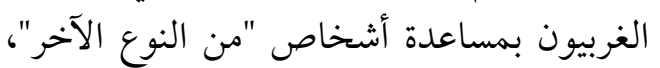

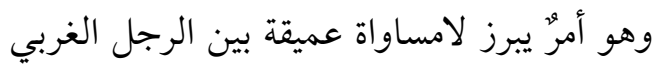

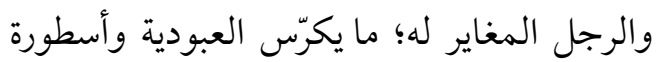

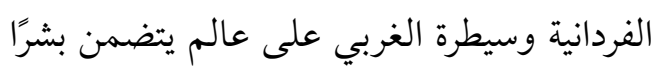
من النوع الآخر.

يشير المؤلّفّ كذلك إلى أنّ الإعلانات الاحتفالية

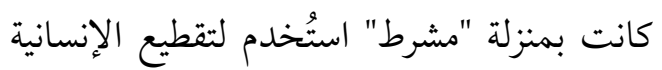

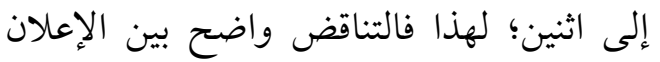
الفرنسي لحقوق الإنسان والمواطن عام لهن 1789
مقدمة

تشكّل العنصرية، بمختلف أنواعها

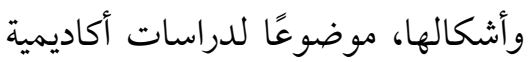
من تخصصات متنوعة ومتقاطعة، بهدف إبراز تجلياتها ومسارها التاريخي والسياسي ووقعهات ومناطيات النفسي والاجتماعي ورموزها الأنثرويولوجية، ولغيانيا

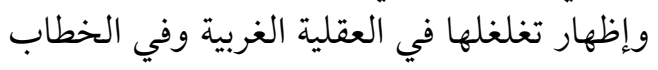

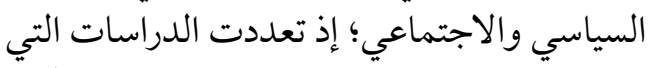

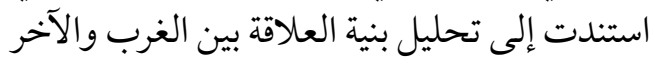
وتفكيكها، مرتكزة على ثنائية "الأنا" و"الآخر"، الألئ

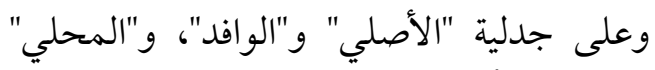

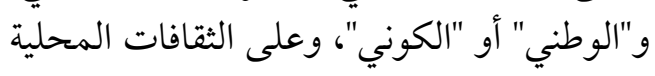

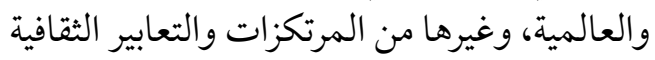

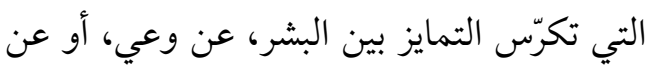

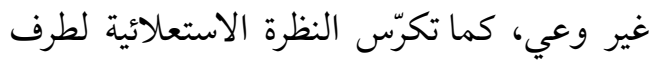
ما تجاه طرف آخر. طرحت العنصرية إشكاليات عديدة من قبيل إلبيل

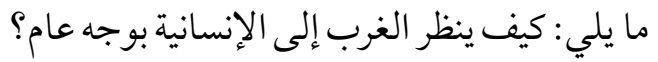

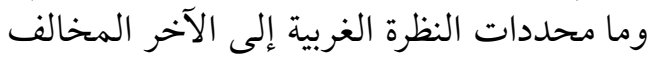

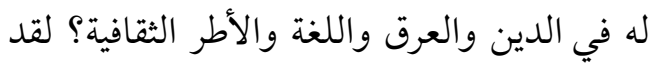

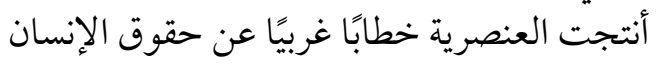

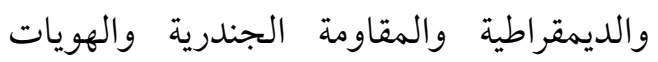

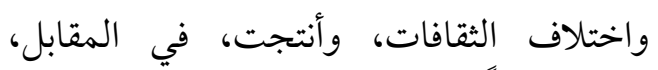

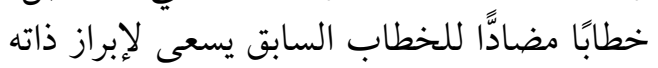

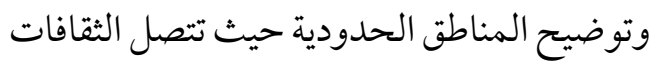

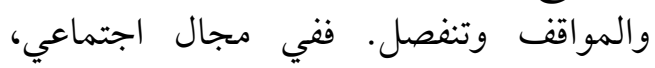

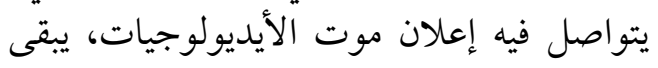
الخط الفاصل بين أنصار العنصرية وأعدائها هو ذلك الانشطار الذي يفصل بين رؤى كونية النصار الفنصرية واعدانيا

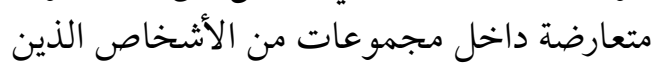
يملكون قواسم مشتركة تشكّل ثقافتهم. وفق هذا السياق، يأتي كتاب الباحث الكندي

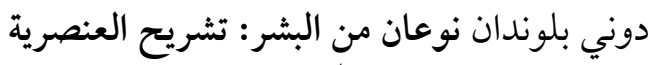

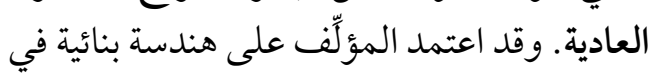


المعروف بـ "أسترالوبيثيكوس أفارينسيس" الانسان، Australopithecus afarensis الإنسان الماهر والإنسان المنتصب، إنسان جاوة

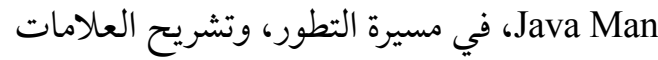
الفارقة وشبكة الرموز التي أنتجتها الإنسانية. لكنه

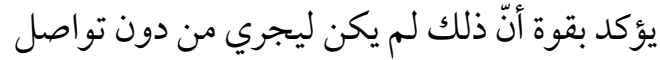

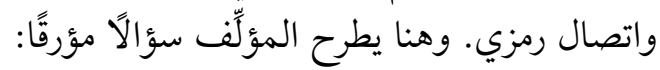

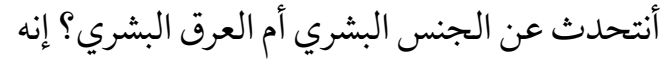

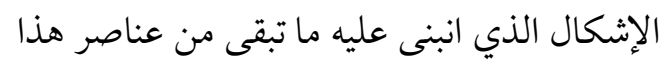
الفصل؛ لهذا عزز أطروحاته بمناقشة كتابات كلود ليفي ستراوس Claude Lévi-Strauss 2009)، ونصوص فولتير Voltaire (1694-1778)، وجان جاك روسو Jean-Jacques Rousseau (1712-1778)، والأبحاث البيولوجية، وغيرها من رون

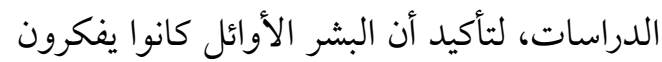

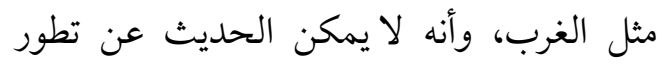

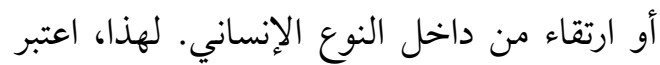

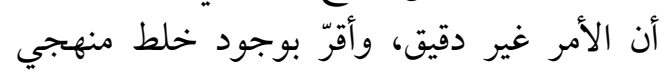
بين مختلف مستويات التصنيف (نوع، جنس،

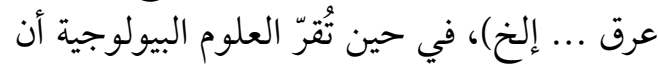
البشر - بالمعنى البيولوجي - يشكلون نوعًا واحدًا،

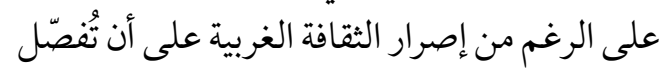

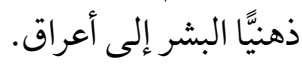

من ناحية أخرى، يناقش الفصل الثالث "انحراف

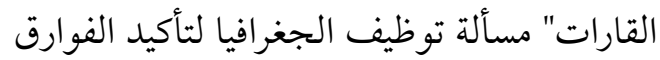
بين البشر؛ فالفرق واضح بين قارات الجغرافيا

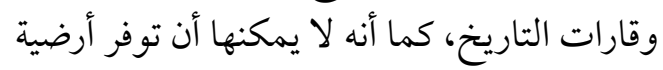

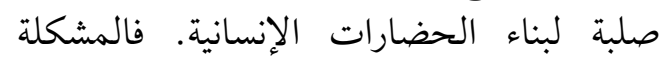

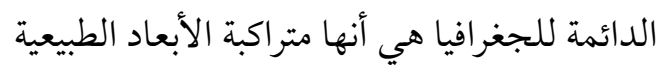

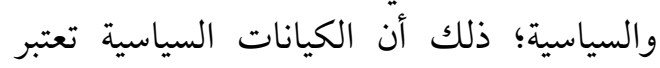
نفسها ظواهر طبيعية من اللحظة التي اختارت

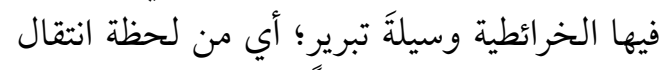

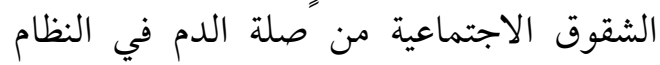

وبيَن انخراط المواطنين الفرنسيين في عملية بيع وشراء العبيد في المستعمرات، لوجود إنقات اعتقاد في اختلاف مفهوم الإنسان عن مفهوم

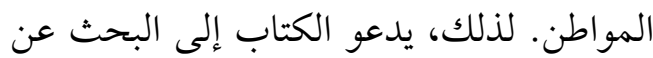
جذور العنصرية بوصفها منظومة أيديولوجية، في الإعلانات النيلة والكبرى عن حقوق الإنسانية، وليست في مؤسسات مثل العبودية أو الحرب؛

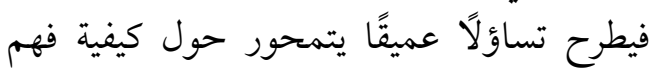
امتناعنا عن إدراك الإنسانية الراهنة باعتبارها

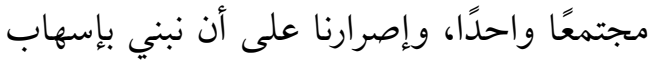
وإطناب صورة عن إنسانيتين متوازيتين. إنه وإِين سؤال مركزي شكّل أطروحة الكتاب المركزية

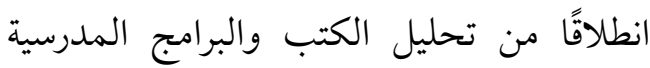
في مقاطعة كيبيك الكندية؛ لأثرها الواضح في تئي تقديم رواية مُركزة عن الثقافة الغربية، وباعتبارها مشكلة لدعامات الثقافة الغربية وعاكسة للإجمماع الاجتماعي. والحال أن بنية البرنامج نفسها تنطلق من الفصل بين النوعين الإنسانين: الإنسانية التاريخية "نحن" والإنسانية الجغرافية "الآخرون". ويناقش الفصل الحاجز الذهني بين الإنسان التاريخي والإنسان الجغرافي، والحدود الصادمة بين النوعين حين تؤكد كتب التاريخ العامّ سلسلة النسب والإنسانية البيضاء، وأثناء كل مرحلة تطورية، حيث يجري تقطيع الغرب على نحو

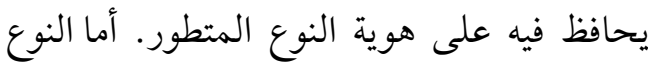
الإنساني الآخر، فيبقى محصورًا في منطقة الظلام

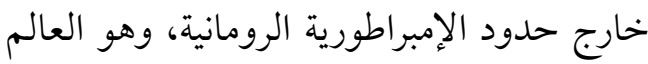
الثالث اليوم. ت الثو

يستند المؤلّف في الفصل الثاني "لوسي القردة" إلى مجموعة من المعطيات الأركيولوجية

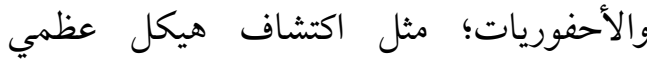

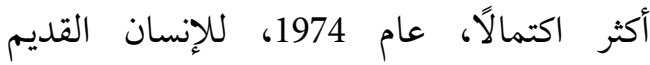


يقدّم الإنسان الغربي على أنه صانع للتاريخ؛

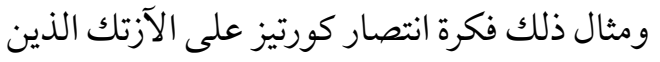

نظروا إلى الإسبان نظرتهم إلى آلهة "بيض" (1).

الواضح أن الثقافات الغربية اعتمدت تصورًا رمزيّا

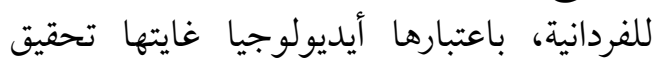

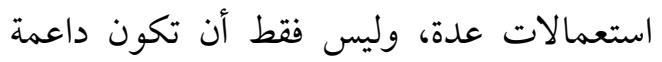

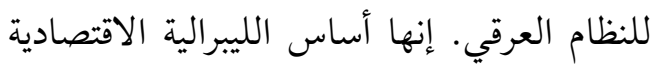

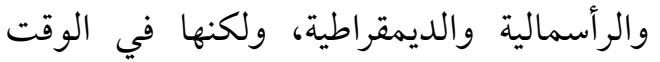
نفسه تشتغل بالتناسب مع النسق العامّ لتصوراتنا، كما تساهم في الفصل بين النوعين البشريين وفي تحويلهما إلى كيانات من طبيعة بين بيولوجية الفين البرين

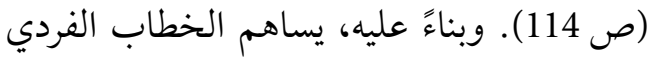

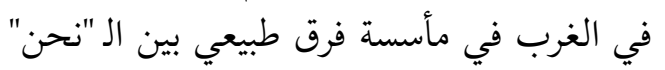

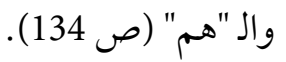

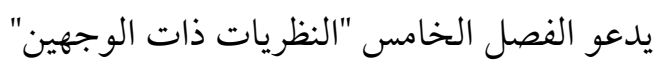

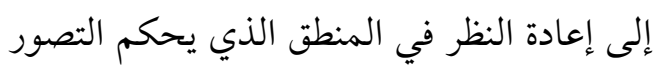

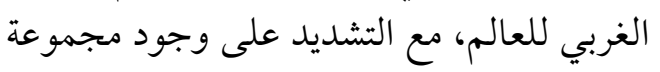

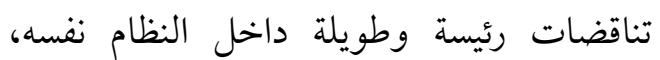

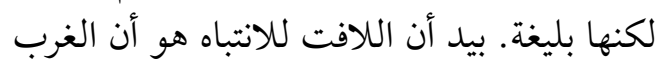

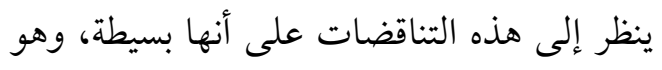

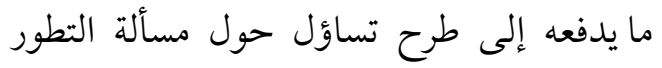
والتخلف: ألايجدر أولاً النظر إلى إلى النوعين

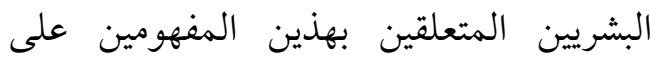

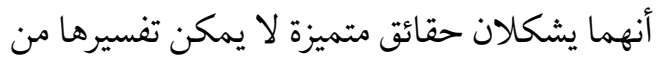

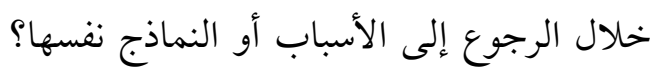

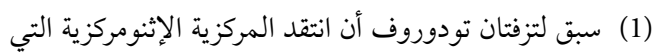

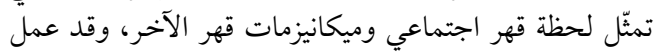

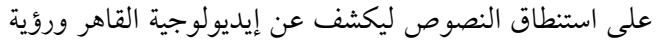

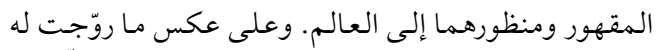

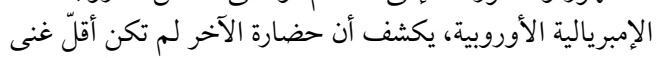

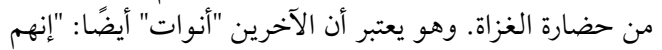

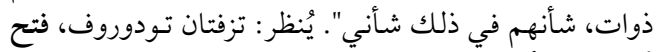

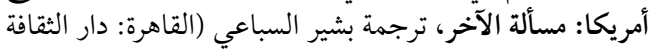

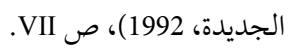

القديم إلى الحدود الإقليمية في المنظومة العالمية

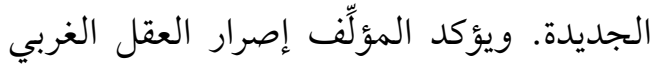

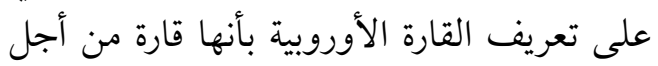

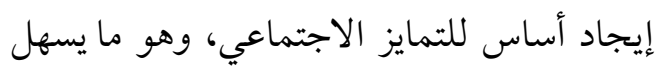
الانتقال من السوسيولوجي إلى البيولوجي. والواقع أن الدراسات المهتمة بعلم الوراثة أكدت أن الأعراق البشرية لم تكن موجودة بصفتها أصنافًا

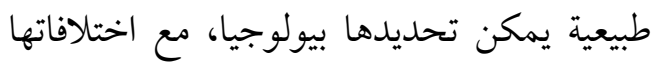

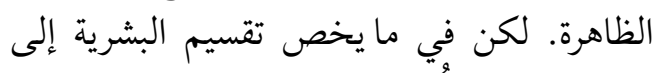

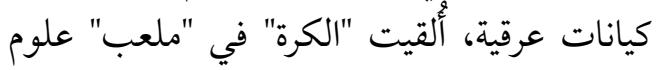

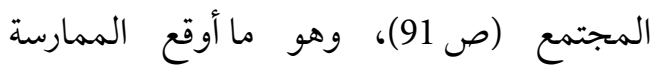
العملية في تناقضات عديدة على مستوى القوانين والإجراءات. ويقدم المؤلّف مثالًا دالَّا على ذلى ذلك الك أنين

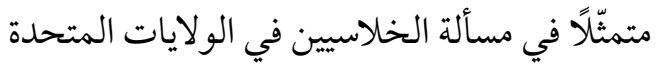

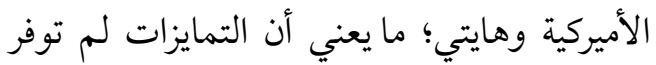

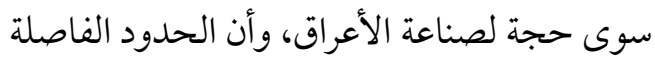
هي حدود اعتباطية مثل الحدود الفاصلة بين الفرافين

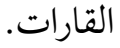

يُستهل الفصل الرابع "كورتيز والآزتك" بتأكيد أنّ

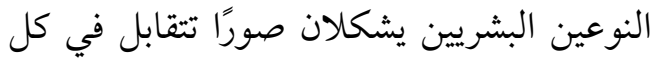

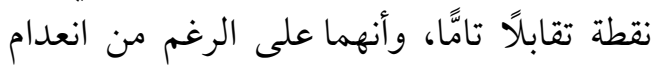

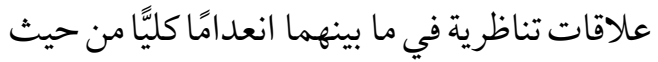

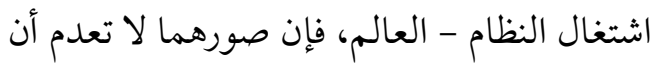

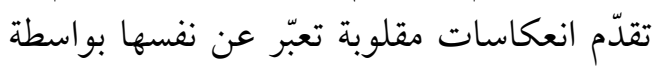

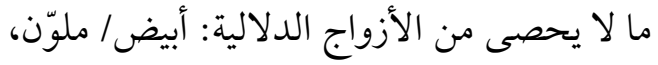

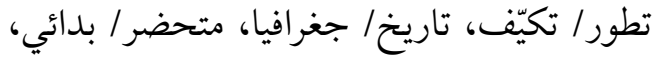

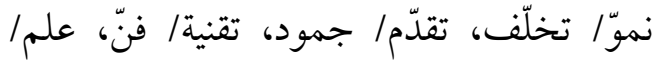

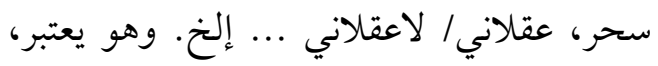

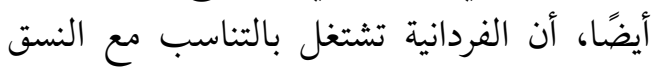
العام للتصورات الغربية، وهي تساهم في الفصل الفئل

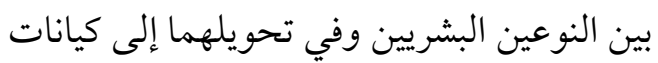
من طبيعة بيولو جية. بَيد أن التصور الذهني الغربي لئي الثي كيات 
يكشف الفصل السادس "الرواية الرسمية" أن النظام

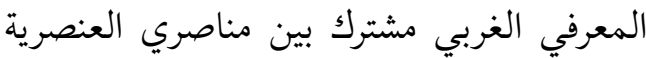
وخصومه، ويرى أنّ لعبة التفسيرات أو النظريات تبقى غير مفهومة من دون العودة إلى التركيبة

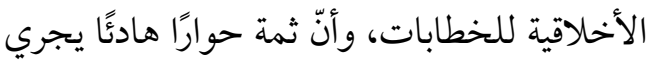
باستمرار بين القلب والعقل، وبين العلم والفكر. فالحوار ممتد بين العلم والأخلاق حول الإنسانية

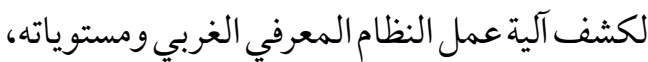

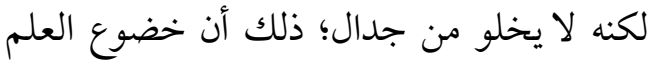
لمصلحة الأخلاقيات يخرج بوضوح من بنية

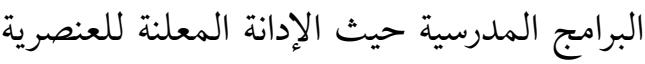

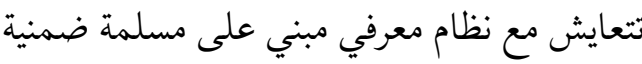
عن وجود اختلاف طبيعي بين النوعين البشريين. السؤال المطروح هو: كيف يمكن أن نكون

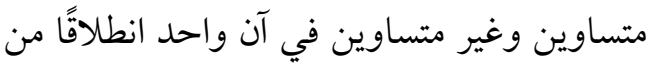
نصوص منظمة الأمم المتحدة للتربية والعلم

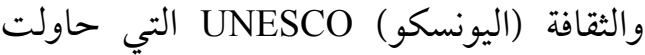

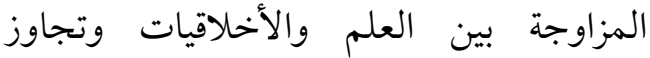
الالتباس في الاستخدامات المعيارية والمعرفية

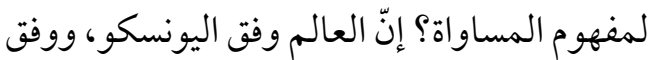

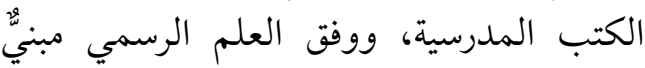

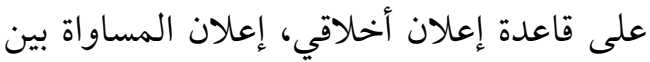

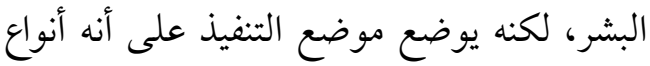

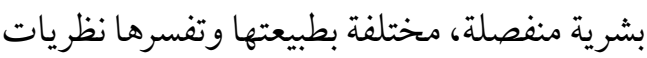
متناقضة: إنه منطق العنصرية (ص 168). تبقى النظرية التطورية قائمة لتبرير المركزية

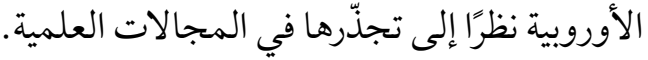

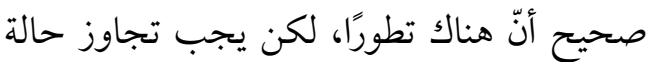

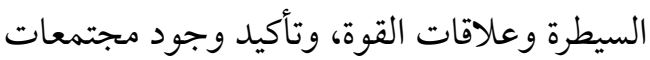
مستقلة أثناء الحديث عن عملية إلغاء الاستعمار

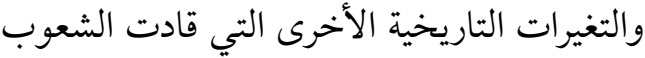

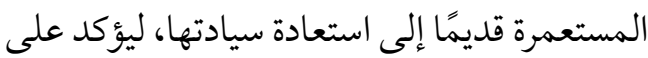

من أجل اكتشاف نظام التناقضات بين الخطابين يجب اعتبار مجمل البشر حقيقة واحدة، وكائنات من الطبيعة نفسها.

فإلى جانب مبدأ التطور والارتقاء، يطرح المؤلّف

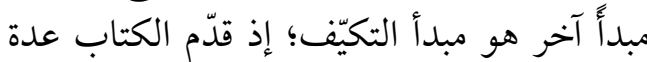
أمثلة متعلّقة بالنظرة المزدوجة للتكيّف فمدا فما يعتبره الغرب بيئة مواتية له للإنتاج والتقدم، يقدّم تفسيرًا

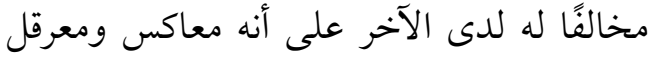

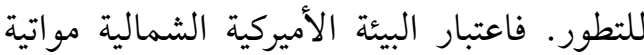

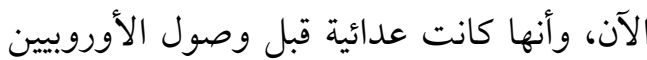

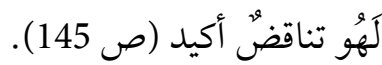

يُفسر الفصل نظرية أرنولد توينبي قائل Toynbee التفاعلات بين البيئة والجماعات البشرية بأن الباتئ

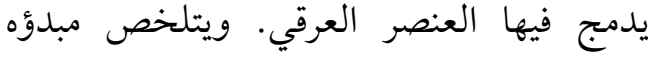

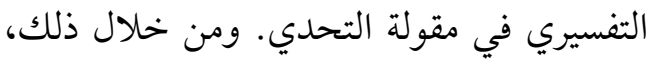
يستطيع الباحث متابعة "اللعبة" البارعة لبناء

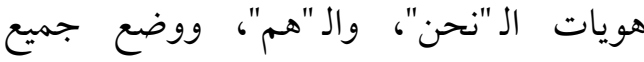

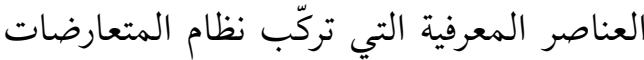

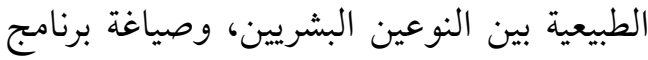

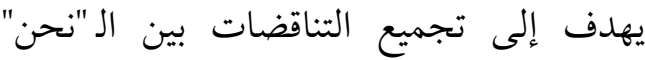
و"الآخرين". ويناقش كذلك نظيع نظرية العزلة؛

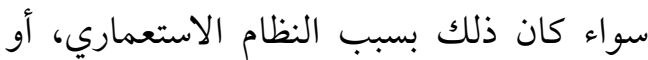
العوامل الطبيعية أو السكانية، أو الدين، أو البنى البعائ

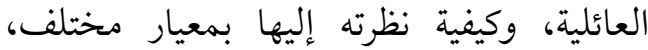

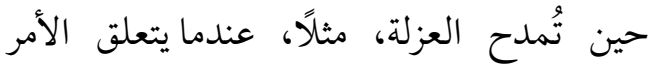
ببريطانيا واليابان، ثمّ كيف أنّ هذه العزلة تصبح مقيتة من خلال التجربة الإندونيسية أو الكوبية. لهذا، يدعو الكتاب إلى نقاش هادئ بين مؤيدي

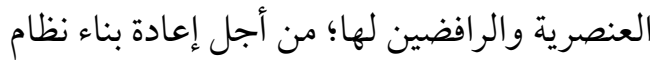

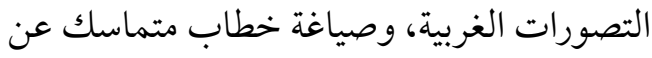

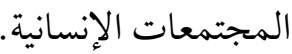


هناك قيم عمومية كونية إلا إذا تأسست داخل بنية اجتماعية عمومية كونية.

يدعو المؤلّف في الفصل الثامن "مبادئ أولية

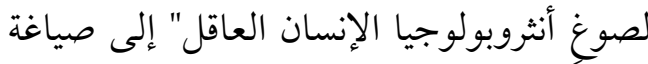

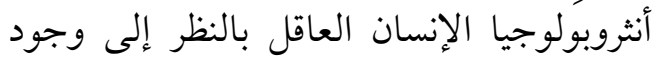

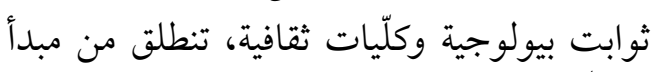

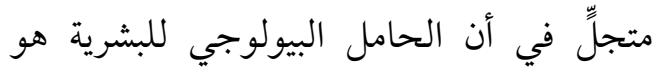

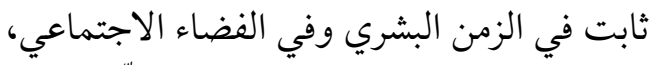

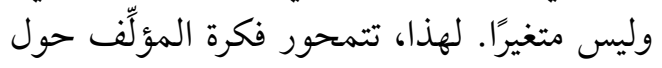

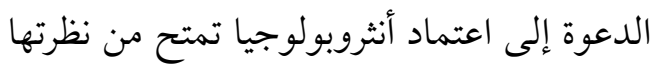

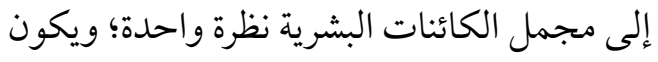

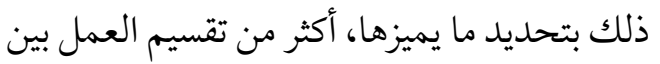

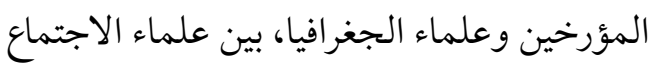

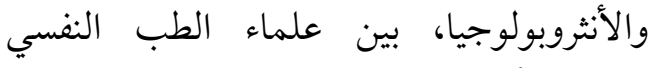

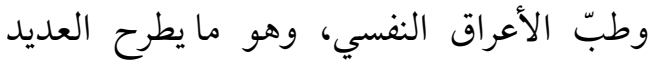

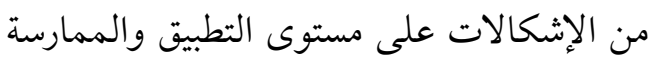

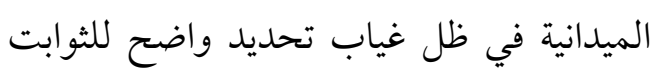

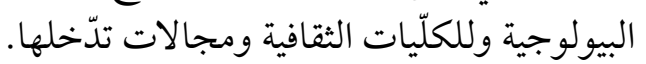

من أجل تجاوز الإشكاليات المطروحة في متن

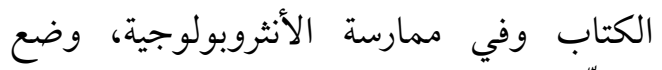

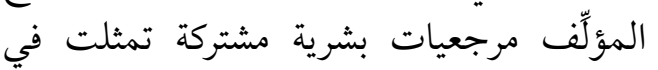
اللغة والفكر وعلم السحر وتأنيس البشر وتشبيك

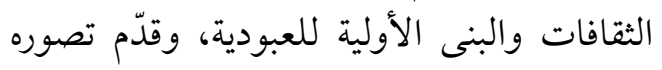
لكل عنصر من هذه العناصر.

\section{ملاحظات نقدية}

بعد أن عرضنا محتوى الكتاب عرضًا مختصرًا، نُبدي الملاحظات التالية.

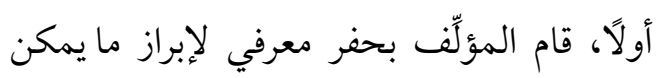

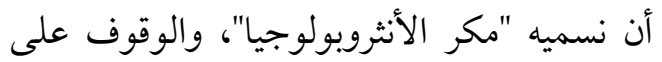

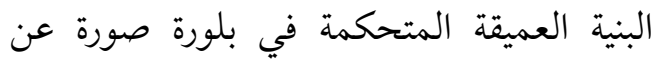
الآخر، فالكتاب يدين ضمنيًّا توظيف الاستعمار

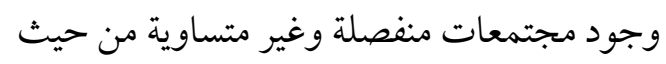
التقدم. لهذا، يستحضر نظرية تنمية التخلف عند مئد

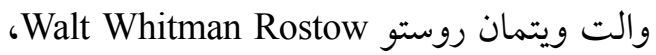
واختلافها عن النزعة التطورية الارتقائية خلال روست القرن التاسع عشر.

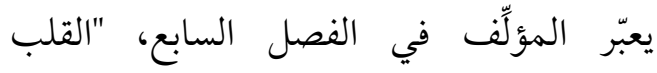
وما يشير إليه"، عن حيرته في الآختيار بين الفين

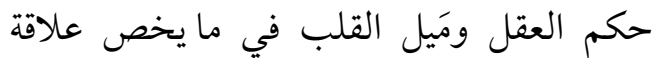

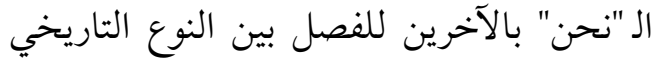

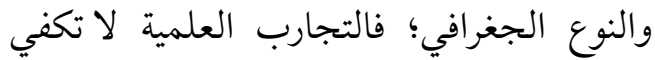

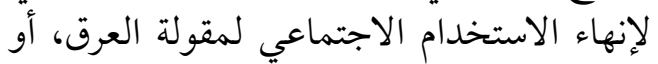

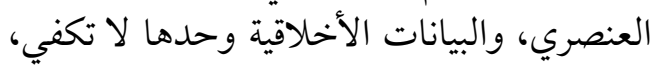

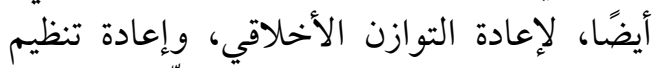

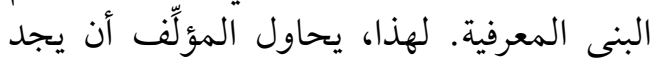

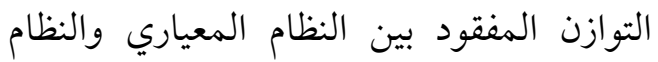

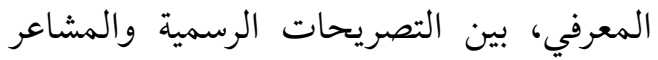

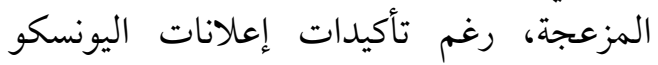

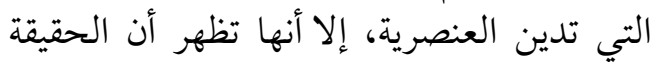

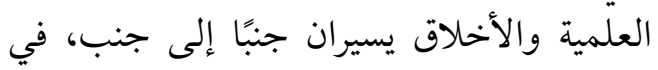

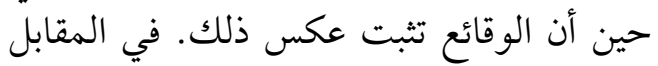

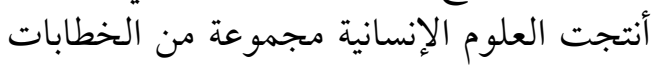

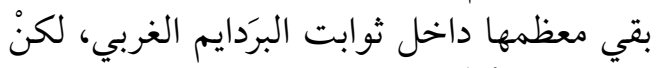

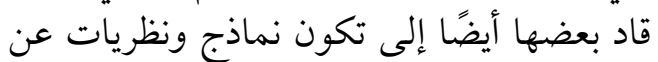
التخلف تأخذ في الحسبان البنى الاجتمان إلماعية

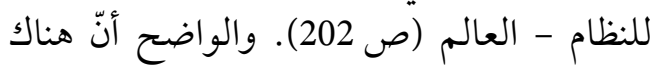

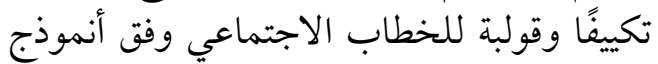

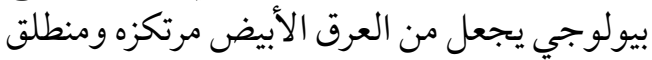

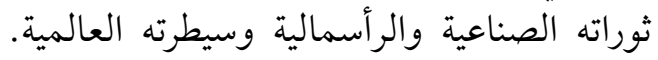

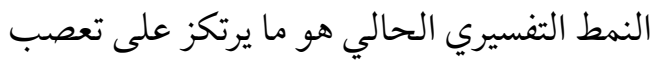

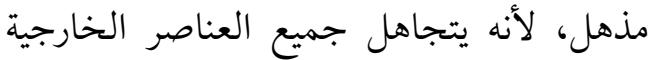

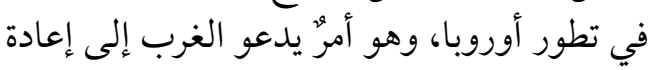

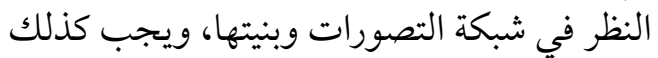

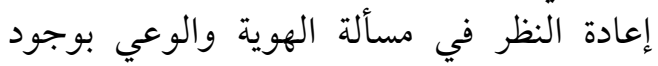
نسبية ثقافية تفرض الحترام الاختلافات الثاتئ الثقافية بين الطبقات الاجتماعية والمناطق. فلن تكون الاخناف الفنافية 
عمومًا، ينسجم طرح المؤلّف مع ما ذهب

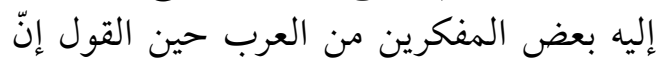

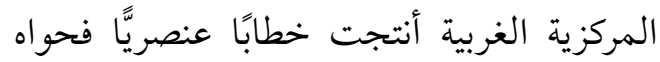

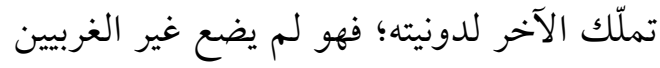
في مستوى رتبة الغربيين، بل حُجزوا في مرتبة فئن الفربين

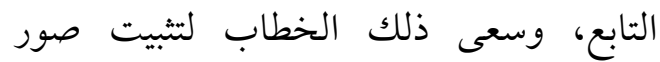

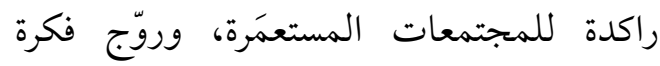

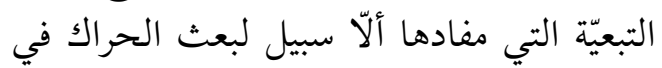

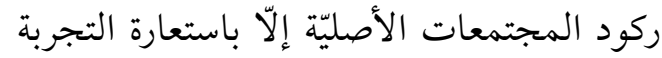

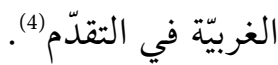

يعتبر الكتاب مساهمة حقيقية وجادّة في رصد

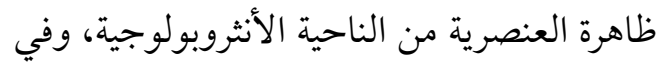
تشريح بنية العقل الغربي في هذه النقطة تحديدًا،

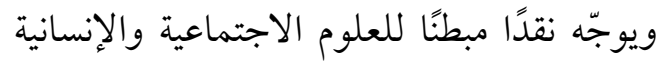

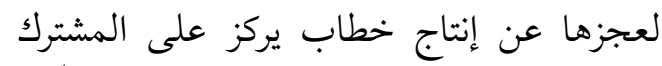

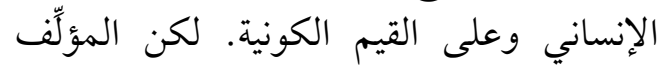

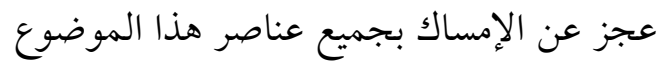
المتعدد المداخل؛ إذ اكتنى بتشريح الظاهرة

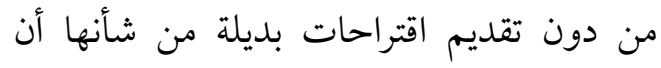

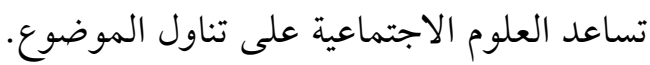

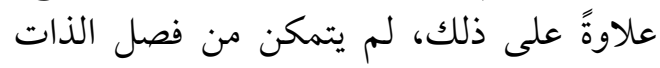

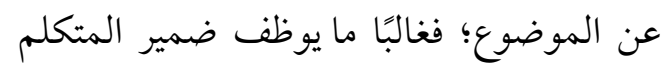

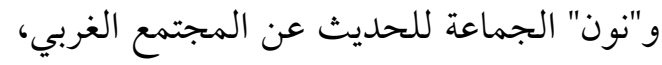

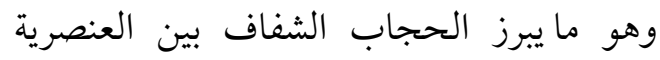

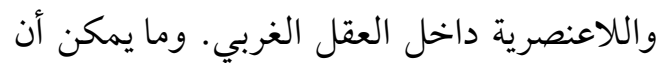

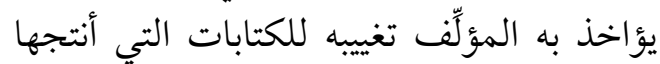

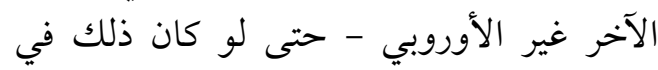

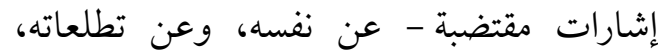

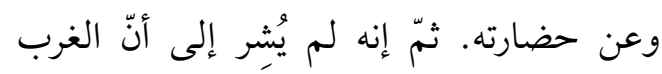

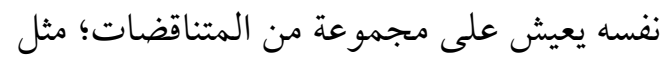

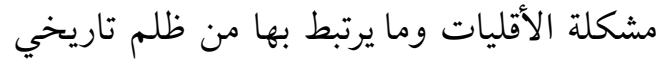

$$
\text { (4) على سبيل المثال، ينظر : المرجع نفسه. }
$$

للأنثروبولوجيا، خاصة بعد أن أثار أنثروبولوجيّو

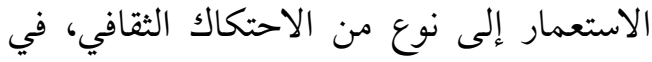

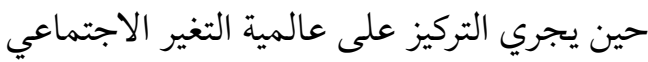

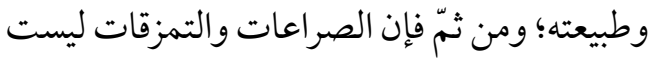

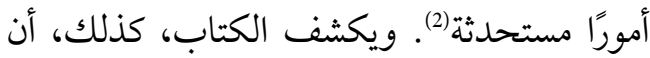

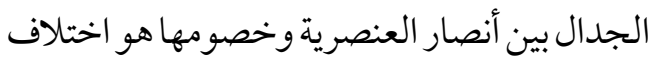
في القيم. ويبيّن أن التفوق العرقي ليس سوى العيد تسمية عنصرية لعبقرية الغرب المسماة غالبًا ذكاءً أو دينامية يمر رها بعض مؤلفي الكتب البع المدرسية، من دون أن يروا في ذلك أدنى فهِم عنصري.

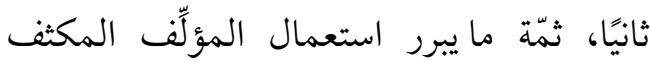

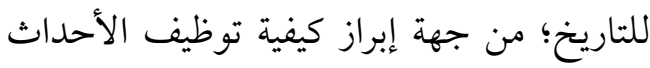
التاريخية لمصلحة القوى الغربية وجعلها مفسرة لكل تطور. فالغرب اخترع كل شيء، الاضطهاد الترجية والديمقراطية، وجلب كل شيء للعالم، الطغيان

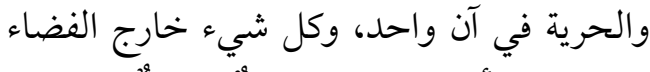

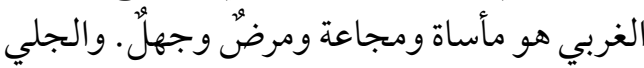

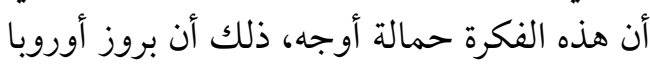

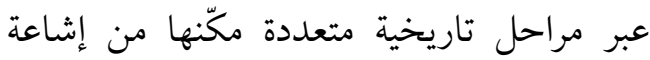
نُظمها الفكرية والثقافية والسياسية والاقتصادية،

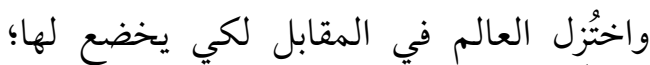

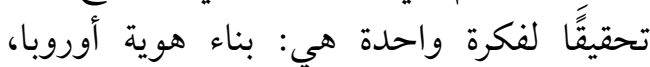

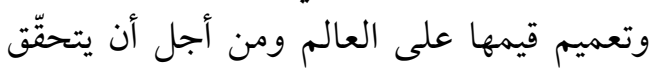
ذلك، يلزم الإجهاز على المكوّنّات الحضارية إنى

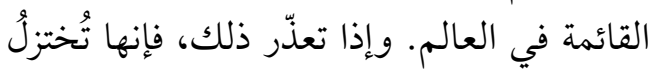

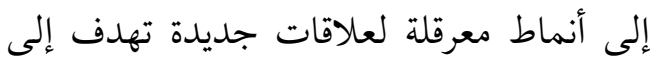
وحدة الإنسان والتاريخ (3). (2) جيرار لكلرك، الأنتروبولوجيا والاستعمار، ترجمة جورج كتورة (بيروت: المؤسسة الجامعية للدراسات والنشر، 1982)، ص صنورة 122. إنكا بهأن هذا النقاش، يُنظر : عبد الله إبراهيم، المركزية الغربية: إشكالية التكون والتمركز حول الذات (بيروت: المركز الثقافي الثرائ العربي 1997). 
قادرة على تجاوز حالة الركود والانحسار في مخرجات الدراسات الغربية. وقد برزت في الآونة

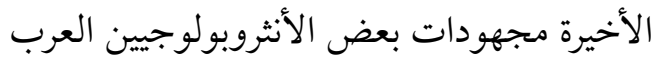
من أمثال عبد الله حمودي في مشروعه المتعلّق

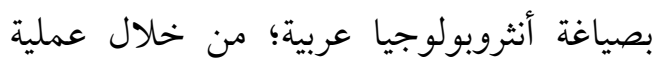

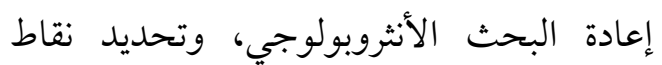
الالتقاء والانفصال بين خطابات الأنثروبولوجيا الكلاسيكية والكولونيالية، وبين وضعية الباحثين العرب وتطلعاتهم في ما يخص إعادة تركيب

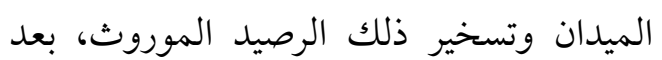

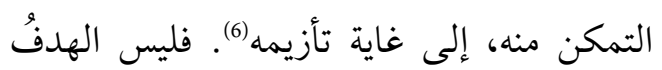
إنتاجَ خطاب طوباوي، بل خلق حوار بين كل الل الأطراف، بعيدًا عن لغة السيطرة والهيمنة، على بلى بلى لئل نحو يكون ملائمًا للبيئة التي أثنتج فيها.

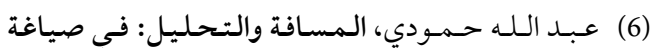

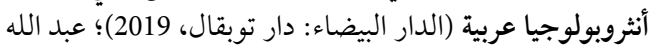

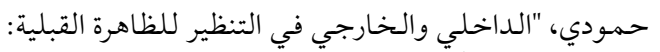

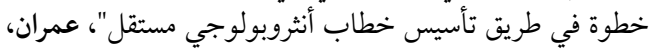

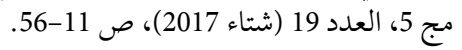

ومعارضة لتبني النماذج الغربية للفدرالية المتعددة القوميات

إن استحضار المؤلّف مسألة العنصرية في الخطاب الغربي استحضارًا واعيًا يبرز النسيج الثقافي والفكري الذي أنتج هذا الخطاب والمؤسسات الداعمة له، وهو مايتجلى في النقاش الدائر في الأوساط الأكاديمية الغربية

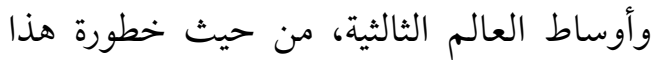
العنف والإقصاء الممارس داخل علاقة غير

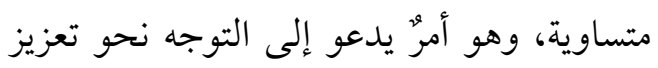

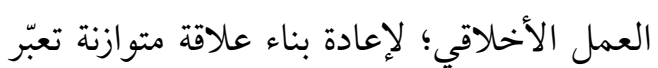

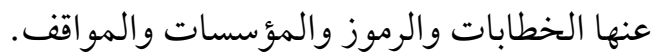
نتطلع إلى أن تنتج دول العالم الثالث معرفة أنثروبولوجية وسياسية لتقديم نفسها، وأن تكون إلى النيج

(5) ويل كيمليكا، ويل كيمليكا، أوديسا التعلدية الثقافية: سبر البرات

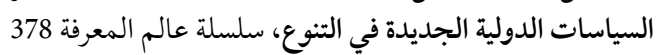

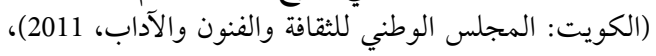

ص 32.

\section{References}

\section{المـراجع}

إبراهيم، عبد الله. المركزية الغربية: إشكالية التكون والتمركز حول الذات. بيروت: المركز الثقافي العربي 1997. تودوروف، تزفتان. فتح أمريكا: مسألة الآخر. ترجمة بشير السباعي. القاهرة: دار الثقافة الجديدة، .1992

حمودي، عبد الله. المسافة والتحليل: في صياغة أنثروبولوجيا عربية. الدار البيضاء: دار توبقال، 2019. .

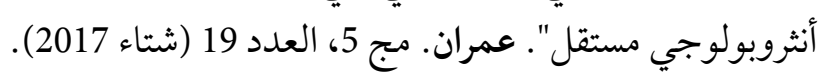

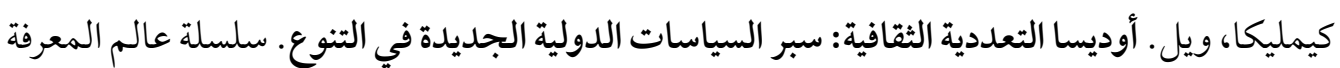

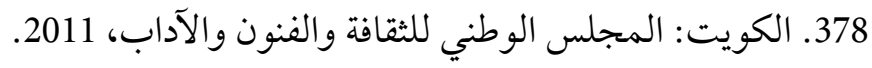
لكلرك، جيرار. الأنتروبولوجيا والاستعمار . ترجمة جورج كتورة. بيروت: المؤسسة الجامعية للدراسات والنشر، 1982. 